\title{
Brown University Oncology Research Group
}

National Cancer Institute

\section{Source}

National Cancer Institute. Brown University Oncology Research Group. NCI Thesaurus.

Code C157456.

A cancer research group created in 1994 to coordinate clinical cancer research for Brown University affiliated hospitals, including Rhode Island Hospital, the Miriam Hospital, Roger Williams Medical Center, Memorial Hospital of Rhode Island, and Women \& Infants Hospital of Rhode Island, and the Alpert Medical School faculty, with the mission of improving cancer care through the implementation of innovative, multidisciplinary cancer clinical trials. 